\title{
Potentiell nosokomiale Ansteckung mit Mycobacterium chimaera
}

\section{Barbara Hasse, in Zusammenarbeit mit der Taskforce M. chimaera*}

PD Dr. med., Klinik für Infektionskrankheiten und Spitalhygiene, UniversitätsSpital Zürich

* Erik C. Böttger, Zürich; Simon Costabile, Zürich; Samuel Erny, Bern; Céline Gardiol, Bern; Achim Häussler, Zürich; Peter Keller, Zürich; Daniel Koch, Bern; Virginie Masserey, Bern; Rafael Moreno, Bern; Hugo Sax, Zürich; Matthias Schlegel, St. Gallen; Bettina Schulthess, Zürich; Rami Sommerstein, Bern; Thomas Suter, Bern; Markus Wälti, Bern; Andreas Widmer, Basel

Wenn Patienten Monate oder Jahre nach Chirurgie am offenen Herzen unklare Infektionen, Blutkultur-negative Endokarditiden oder Symptome einer Systemerkrankung entwickeln, dann ist es wichtig, eine nosokomiale Infektion mit dem langsam wachsenden, nichttuberkulösen Mycobacterium chimaera in Erwägung zu ziehen und zeitgerecht abzuklären. Eine Checkliste fasst zusammen, bei welchen Situationen Abklärungen empfohlen werden.

\section{Ausgangslage}

Am UniversitätsSpital Zürich (USZ) und an den Universitätsspitälern Basel und Liestal (USB) wurde bei bisher zehn Patienten im Zusammenhang mit einer Herzoperation eine schwerwiegende und disseminierte Infektion mit Mycobacterium chimaera diagnostiziert. Dieses nichttuberkulöse Mykobakterium wurde wahrscheinlich während des Eingriffs aus dem Wasser von Temperaturregulierungsgeräten der Herzlungenmaschine der Firma Sorin (jetzt LivaNova) vom Typ 3T (Heater Cooler Devices, HCDs) via Aerosole durch die Luft ins Operationsgebiet übertragen [1]. Das Bundesamt für Gesundheit (BAG) und Swissmedic haben die Öffentlichkeit und die Spitäler am 14. Juli 2014 darüber informiert [2, 3]. Inzwischen wurden weitere kontaminierte HCDs in der Schweiz und in anderen Ländern gefunden [4].

Die LivaNova 3T HCDs wurden entweder in der Fabrik oder in den Spitälern aus dem Leitungswasser kontaminiert. Weltweit wurden mehr als 70 mit M. chimaera infizierte Patienten diagnostiziert. Es ist möglich, dass es (auch in der Schweiz) weitere mit M. chimaera infizierte, aber noch nicht diagnostizierte Patienten gibt. Die Diagnose ist schwierig zu stellen, da die ersten Symptome unspezifisch sind, das Bakterium schwierig zu kultivieren ist und die Inkubationszeit bis zu 5 Jahren dauert. Viele dieser Fälle wurden in erster Instanz als fever of unknown origin (FUO) abgeklärt und einige als Sarkoidose fehldiagnostiziert und mit Steroiden behandelt. Schwierig ist auch, dass oft extrakardiale
Manifestationen der Erkrankung der kardialen Manifestation vorauseilen (Knocheninfekte, Panzytopenie, Chorioretinitis, Leber- und Nierenbefall).

Dieses Schreiben soll helfen, dass die betroffenen $\mathrm{Pa}$ tienten so früh wie möglich erkannt werden, da fortgeschrittene Fälle trotz korrekter Antibiotikatherapie letal verlaufen sind. Da Patienten möglicherweise nur mit einer raschen Reoperation geheilt werden können, wäre eine Frühdiagnose wichtig.

\section{Mycobacterium chimaera, was ist das?}

M. chimaera, ein langsam wachsendes, biofilm-bildendes, nichttuberkulöses Mykobakterium, wurde 2004 aus dem Mycobacterium avium-Komplex ausgesondert und wird ohne Zusatzuntersuchungen mit diesen Bakterien verwechselt. Sehr selten kann es bei immunsupprimierten Patienten eine Pneumonie verursachen. Dass es auch Endokarditiden, Gefässprotheseninfektionen und disseminierte Infektionen hervorrufen kann, wurde erst im Zusammenhang mit diesem Ausbruch bekannt.

\section{Wann muss an eine $M$. chimaera-Infektion gedacht werden?}

Der Verdacht auf eine M.chimaera-Infektion sollte nach heutigem Stand des Wissens bei allen Patienten mit unklaren Infektionen oder Systemerkrankungen nach einer Operation an der Herz-Lungen-Maschine erwogen werden. Betroffen sind vor allem Patienten mit einer künstlichen Herzklappe, mit einer Ge- 
fässprothese oder mit kreislaufunterstützenden intrakardialen Geräten. Es wurden aber auch selten M. chimaera-Infektionen bei Patienten nach Operation mit HCDs ohne Implantation von Fremdmaterial beschrieben (Bypassoperation). Noch unsicher ist der Zusammenhang mit kontaminierten HCDs bei Lungentransplantation.

\section{Konkrete Massnahmen}

Es ist davon auszugehen, dass die Infektion nur vereinzelte Patienten betrifft. Die negative Voraussagekraft der diagnostischen Verfahren in der Latenzphase ist tief. Eine breit angelegte Untersuchung aller Patienten ist deshalb nicht sinnvoll. Bei bestimmten Infektionstypen (siehe unten), bei Fieber unklarer Ursache oder einer unklaren systemischen Erkrankung bei Patienten nach Operation mit HCDs auch Jahre zuvor muss diese Diagnose ausgeschlossen werden - insbesondere, wenn bei Endokarditis-Verdacht die konventionellen Blutkulturen negativ bleiben.

\section{Checkliste: Wann ist M. chimaera-Screening/ -Diagnostik sinnvoll?}

Trifft das Expositionskriterium zu und ist einer oder mehrere der untenstehenden Punkte erfüllt, wird empfohlen, diese Patienten für die Abklärung an ein infektiologisches Zentrum zu überweisen.

Korrespondenz

PD Dr. med. B. Hasse Klinik für Infektionskrankheiten und Spitalhygiene UniversitätsSpital Zürich Rämistrasse 100 CH-8091 Zürich Tel. 0442559237 barbara.hasse[at]usz.ch
Herzchirurgie mit extrakorporalem Kreislauf und Verwendung von Heater Cooler Devices (Kunstklappen,

o Gefässprothesen, Herztransplantation, Einbau Ventrikuläres Unterstützungssystem, Bypassoperation) in jedem Schweizer Spital

und einer/mehrere der folgenden Punkte

\begin{tabular}{ll}
\hline o & Kultur-negative Prothesenklappenendokarditis \\
\hline o & Kultur-negative thorakale Aortenprotheseninfektion \\
\hline o & Kultur-negative Sternuminfektion und/oder Mediastinitis \\
\hline o & Fieber unklarer Ursache oder Vaskulitis \\
\hline o & Unklare Systemerkrankung \\
\hline o & Sarkoidose oder andere granulomatöse Erkrankung
\end{tabular}

\section{Empfohlene Abklärungen in der Praxis}

- Anamnese (Fieber, Müdigkeit, Gewichtsverlust, Nachtschweiss)

- Körperliche Untersuchung

- Labor (Blutsenkungsreaktion, C-reaktives Protein, Blutbild, L-Lactatdehydrogenase, Transaminasen, Kreatinin)

- Blutkulturen

\section{Weiterführende Abklärungen am Zentrum}

- Echokardiographie

- Histopathologie bei Reoperation / Herz-Biopsie

- Mykobakterien Kulturen/PCR:

- Mykobakterien Blutkulturen (BacTec myco Lytic/F bottles [BD Bioscience])

- Mykobakterien Kulturen oder Mykobakterien PCR bei Reoperation/Biopsie

\section{Weiterführende Auskünfte}

Bundesamt für Gesundheit BAG

Abteilung Übertragbare Krankheiten

Tel. +41 (0)584638706

epi[at]bag.admin.ch

\section{Literatur}

1 Sax H, Bloemberg G, Hasse B, et al. Prolonged Outbreak of Mycobacterium chimaera Infection After Open-Chest Heart Surgery. Clinical infectious diseases: an official publication of the Infectious Diseases Society of America 2015;61(1):67-75.

2 Massnahmen für höhere Patientensicherheit in der Herzchirurgie. Bundesamt für Gesundheit (14.07.2014). https://www.news.admin. $\mathrm{ch} /$ message/index.html?lang=de\& msg-id=53774

3 Hypo-/Hyperthermiegeräte (Heater Cooler Units, HCU) für die Herzchirurgie - neue Empfehlungen: Mögliche Beeinträchtigung von Oxygenatoren. Swissmedic (21.06.2016). Hypo-/Hyperthermiegeräte (Heater Cooler Units, HCU) für die Herzchirurgie - neue Empfehlungen - Swissmedic.

4 Risk assessment on Mycobacterium chimaera infections associated with heater-cooler units. ECDC (18.11.2016). http://ecdc. europa.eu/en/publications/Publications/RRA-mycobacteriumchimaera-November-2016.pdf 